\title{
Representaciones, identidad y prácticas discursivas de los espacios de frontera nicaragüense (Costa Rica-Nicaragua)
}

Miguel Ayerdis

Recepción: 06-07-15/Aceptación: 12-12-15

\section{Resumen}

Este trabajo tiene un carácter provisional en su temática dado que busca teorizar con el fin de poner en perspectiva aspectos relacionados a las representaciones, identidades y prácticas discursivas en espacios de frontera. El nivel de profundidad de esta investigación es exploratorio al carecerse en Nicaragua de una base de estudio sólida sobre estos espacios.

Se hizo una revisión bibliográfica relacionada al tema, todas ellas de experiencias latinoamericanas, cuyo hallazgo reviste singular importancia. Por un lado -como se verá en el estudio - los temas de frontera encontrados, cruzan de manera transversal, las prácticas cotidianas, las identidades construidas, las representaciones y los discursos diseñados desde los círculos de poder. Por el otro, la experiencia empírica expresada en algunos estudios, reflejan incoherencias (descuido) en políticas públicas de los gobiernos, producto de desconocimiento del tema y de la actual realidad.

El trabajo que se presenta, aborda de manera general, las anteriores preocupaciones, dialogando con el proyecto de investigación sobre temas fronterizos y transfronterizos que un equipo de la Facultad de Humanidades y Ciencias Jurídicas de la UNAN-Managua realiza en la actualidad y del cual forma parte el autor. En este sentido, el trabajo recoge la experiencia de una primera visita de campo a las comunidades del municipio de Cárdenas, permitiendo tener contacto con los habitantes y recoger información esencial para la elaboración de esta primera aproximación y su condición fronteriza. Son apenas notas y reflexiones de aspectos a seguir profundizando.

Palabras claves: Espacios fronterizos, identidades, representaciones, prácticas discursivas, publicaciones periódicas.

\footnotetext{
Abstract.

This work is an exploratory research that theorizes aspects related to representations, identities and discursive practices in border areas, because Nicaragua lacks of a solid base study in these areas.

A literature review of Latin American experiences related to the subject was carried out with finding singular importance - on the one hand-as results will show-border issues found, transversely crossing the daily practices of constructed identities, representations and speeches designed from the circles of power. On the other hand, the empirical experience expressed in some studies, could reflect inconsistencies in public policies of governments, as a product of lack of subject and current reality knowledge.

This paper addresses in general topics of the research project on border issues that is carried out by the Faculty of Humanities and Juridical Sciences of the National Autonomous University of Nicaragua (UNAN - Managua)
}

Keywords: border areas, identities, representations, discursive practices, newspaper publication. 


\section{Introducción}

La literatura relacionada con los estudios de frontera es variada y diversa. Un recorrido sucinto por algunos estudios en América Latina dan cuenta de preocupaciones académicas que van desde la caracterización histórica y sociocultural de las relaciones fronterizas en regiones del Continente (Arias Olave, 2013), infraestructura fronteriza, ciudades de frontera (analysis, Mendoza Pérez, 2007); redes, mercados (Vargas Hernández, 2005), hasta medios de comunicación y representaciones culturales (Silva Echeto, 2011), para mencionar algunos estudios revisados para el presente trabajo.

De igual manera convergen otros tema de relevancia en los estudios de frontera de mucho interés dentro del ámbito académico, como es lo relacionado con lo nacional y lo transfronterizo. En este ámbito temático ya clásico, se problematiza sobre los estados nacionales, las migraciones, las relaciones sociales y los espacios sociales y culturales, físico o simbólicos.

Uno de los aspectos que más se destacan en estudios con temas que abordan lo nacional y su relación con las fronteras, es el cuestionamiento al estado como locus o percepción que tienen de esta construcción histórica, en contraposición con la visión transnacional. Esta última perspectiva de estudio --visión en boga hoy día-- hace referencia a los tratados de unión y/o de cooperación regional (Caballero Santos y Tabernero Martin, 2014) así como las dificultades a que se enfrentan dentro del acelerado proceso de globalización y del incremento en la movilidad social y la transferencia cultural.

La revisión bibliográfica infiere una preocupación temática recurrente sobre los espacios de frontera, donde el sujeto que vive en estos lugares, es estudiado bajo la premisa de su condición (o no) de ciudadano con derechos y la actuación del estado y las estrategias de sobrevivencia o de arraigo en estos sitios. Las fuentes hemerográficas (publicaciones periódicas) construyen estereotipos del sujeto de frontera diferente al de la vida cotidiana, deformando la realidad y las particularidades de estos sitios. Este último aspecto ha abierto una línea de investigación académica, donde se problematiza acerca de la conflictividad en las representaciones e imaginario social y simbólico del sujeto de frontera y de su espacio sociocultural dentro del que se mueve (Martínez Sergio, 2011).

Es imperativo para cualquier trabajo sobre la temática de frontera el establecimiento de los límites del concepto para ubicar el estudio. Como bien dice Mejía, Fermín de Mejía y Alcántara (2013) al hablar de frontera se debe de tomar en cuenta dos aspectos: el espacio y los límites que recortan, dado que “...permiten definir el área de extensión de la estructura" además -continúa señalando--, "...por el estudio de las densidades de las poblaciones y de la intensidad de los fenómenos que constituyen el corolario de estudio de los límites" (p.70).

Algo importante que Mejía (2013, et.al.) destacan, lo esencial de la frontera y sus límites, es "su función", la cual -agregan los autores - "es de carácter esencialmente geográfico" (p.71). Otro elemento a rescatar de este estudio, es la referencia a concepto de "extensión espacial de la frontera", hasta donde se puede decir que estamos en un espacio fronterizo, sabiendo que es adyacente a otro. Esta extensión --señalan-está relacionadas a la "intensidad de las relaciones que se producen entre espacios colindantes bajo distintas jurisdicciones" (p.71-72).

De lo anterior, se puede decir que los espacios fronterizos son aquellos donde se articulan relaciones sociales, económicas y donde confluyen prácticas culturales diversas. Espacios estigmatizados por un discurso tradicional, construido desde los centros de poder central (las capitales o distritos 
administrativos del Estado), que ve en estos, "los límites de la soberanía nacional", y "la anarquía" política y social (Granados, 1997).

En la prensa periódica, medios televisivos y en la literatura de ficción, se reproducen los estereotipos acerca de los espacios urbanos fronterizos bajo criterios o enfoques cuestionables. La mayor parte de ellos están basados en tópicos cargados de prejuicios y desconocimiento de estos lugares. Como bien lo señala en su estudio Sergio M. Martínez (2011), cuando analiza dos obras donde se representan la ciudad de Tijuana en la frontera mexicana-estadounidense (Santitos y Peregrinos de Aztlán) ofreciendo:

...imágenes estereotipadas de la ciudad fronteriza, espacio en que reina el caos y la prostitución, notamos que la intención de cada narrador difiere en algunos aspectos importantes como son el nivel de conocimiento del espacio referido, la relación en cuanto a dicho espacio y la intención... (p 95)

El propósito de este trabajo,--en general- es contribuir al tema de la frontera, teniendo como marco de discusión los estudios iniciales que la UNAN Managua por medio de la Facultad de Humanidades y Ciencias Jurídicas realiza, sobre los municipios de Cárdenas y San Juan del Sur. Vale decir, que el proyecto de los estudios de frontera (conocido como "proyecto binacional"), implica el trabajo bajo el enfoque (si se le puede llamar así) "Transfronterizo" en el que también participa, --dentro de los convenios establecidos entre universidades--, la UNED (Universidad Nacional a Distancia) de Costa Rica. El proyecto se titula: "Integración no formal o real: territorio transfronterizo Provincia Guanacaste (Costa Rica) y Departamento de Rivas (Nicaragua)".

De manera específica, este estudio de frontera busca una aproximación a la comprensión de la auto referencialidad de los habitantes del municipio de Cárdenas, su condición de vida en espacios de frontera en contraposición a las prácticas discursivas construida en los centros urbanos de poder. Estos pobladores son representados por un lado, como sujeto de frontera "descentrado", sin los atributos conferidos por el Estado-nación estructurado en el siglo XIX (p. 76 Bello de Arellano, y Vilera de jirón, 2000) por el otro, como sujeto "peligroso" que vive en unos límites donde se dirimen y/o defiende la soberanía de los estados, dentro de una lógica moderna.

Es la construcción discursiva, representada por los medios de comunicación de los centros políticos del país, la que se estudia, teniendo presente la auto referencialidad de los habitantes de las comunidades (individual y colectivo) y de su espacio donde se desarrollan y/o construyen relaciones sociales y culturales compleja. Esta lógica identitaria y de integración, negada o menospreciada por el discurso mediático e intereses económicos, no ha podido ser superada, pese los esfuerzos de políticas públicas, dentro de criterio de soberanía nacional (factor contradictorio), realizados por el Estado y el gobierno del Presidente Ortega, prevaleciendo entre la mayoría de los habitantes del interior del país, la imagen de lugares de "conflicto".

\section{Contexto: Proyecto binacional y primer contacto con Cárdenas}

Para comenzar, es bueno recordar que una de las actividades realizadas por la Comisión del proyecto "binacional" de la Facultad de Humanidades y Ciencias Jurídicas, fue la definición, selección y elaboración de los instrumentos de trabajo para el acopio de la información requerida para el estudio. En este sentido, se organizó una visita al municipio de Cárdenas (8 y 9 junio del 2015), uno de los espacios socio-culturales en estudio, con la finalidad de validar los instrumentos a utilizar durante los estudios de campo, tanto en este municipio como en el de San Juan del Sur.

En calidad de miembro de la comisión se aprovechó esta gira de campo para 
conocer las comunidades del municipio de Cárdenas, aplicando técnicas de observación y realizando breves entrevista a pobladores residentes en ocho de las veinticuatro comunidades que conforman el municipio. Este contacto de dos días con pobladores de las comunidades que conforman el municipio fronterizo, permitió la recopilación de información actualizada, acompañando el estudio con entrevistas y de contacto con sus pobladores, las que luego fue analizada y procesada para apoyar la labor bibliográfica o documental que acompaña al trabajo.

El municipio de Cárdenas, perteneciente al Departamento de Rivas, está ubicado en la frontera sur de Nicaragua, colindante al Cantón de Santa Cecilia, República de Costa Rica. Al norte limita con el Lago Cocibolca, al este con el municipio de San Carlos y al oeste con Rivas y San Juan del sur. Su extensión territorial es de 226.63 kilómetros cuadrados. Su población es relativamente pequeña, apenas supera los 7000 habitantes y su vida productiva, social y cultural, está marcada por su condición rural (datos de la Alcaldía del poder ciudadano de Cárdenas). La cabecera del municipio, es la única comunidad que muestras rasgos urbanos y prácticas de vida similares a las ciudades principales del país.

Es pertinente destacar que esta investigación recoge resultados iniciales sobre temas culturales que midan los gustos, las identidades, prácticas discursivas y las representaciones que el resto de habitantes del país tienen de estos espacios y de los sujetos de frontera. Tampoco se tienen estudios acerca de las implicaciones de las mediaciones discursivas de las publicaciones periódicas (y medios audiovisuales) en la construcción simbólica y conceptual de estos sitios, como se explica de manera preliminar en este estudio, cargadas de tópicos $\mathrm{y} / \mathrm{o}$ estereotipos que desfiguran la realidad.

Más allá de un análisis discursivo, desde el punto de vista histórico cultural, la construcción simbólica de los sitios de frontera y su realidad compleja, refleja intereses particulares, pugnas de poder y una visión atrasada sobre el tema, cuyo anclaje de análisis parte de la concepción del estadonación del siglo XIX. En este sentido, los casos aludidos en el estudio, recogen la experiencia vivida en las comunidades de Colón, Mena, Tirurí, El Tablón, Los Angeles, Zapotillo y Peñas Blanca. No se contempla la cabecera municipal del mismo nombre (Cárdenas), por las particularidades excepcionales de su desarrollo, las condiciones de vida y de infraestructura, diferentes al del resto de comunidades.

\section{Hallazgos}

Lo sucedido en junio de 1998 cuando un grupo de ciudadanos de las comunidades del municipio Cárdenas, dieron a conocer a periodistas su deseo de anexarse a Costa Rica, argumentando el grado de abandono de los gobiernos neoliberales (1990-2006), estremeció la opinión pública nacional. En esa época, la reacción de la clase política en el poder fue la de una reacción tradicional, de defensa de la soberanía, suponiendo a priori que se debía a una pretensión histórica expansionista de nuestro vecino y no a faltas de política públicas para esta zona deprimida y hambrienta de esa época. (La Nación digital, http://wvw.nacion.com/In_ee/1998/junio/15/ ultima7.html. Consultado, $\overline{17}$ de agosto 2015)

El anterior ejemplo, representa un punto de inflexión en la historia reciente del municipio de Cárdenas, porque con esta acción, sus líderes buscaban hacerse escuchar. La estrategia, surtió efecto en parte, el gobierno de Arnoldo Alemán (1997-2001) y luego el de Enrique Bolaños (2002-2006), establecieron algunas políticas paliativas (asistencialista) de atención a la población. Durante la visita de campo realizada en junio de 2015, algunos pobladores recordaron ese episodio, asegurando que luego de la denuncia de los pobladores de su abandono, el gobierno construyó algunas escuelas y mejoró los caminos cercanos a la cabecera municipal. 


\section{Retrato autorreferencial de las comunidades en estudio}

A casi dos décadas de esos episodios, la visita a estas comunidades evidenció que sus habitantes consideran esa acción colectiva, comunitaria, como legítima dentro de sus esfuerzos reivindicativos en su condición de ciudadanos con derechos. Acción reivindicativa incorporada a la historia de un pasado reciente, aún presenten en la memoria colectiva de sus protagonistas y las jóvenes generaciones que poco a poco van superando el estado de pesimismo, de angustia y desesperanza en que vivían.

El contacto con sus habitantes también evidenció que la estrategia de "anexión" al vecino del sur en 1998, fue más que todo de protesta ante el estado nacional y no un deseo expreso de separarse del país. Durante las entrevistas realizadas a los pobladores, todos aseguraron que nunca se les ha pasado en la mente, migrar por tiempo indefinido, ni mucho menos dejar el vínculo histórico y cultural del que consideran su país de origen. Ellos consideran que su vida social, cultural y su identidad están vinculadas al lago y a Rivas. Aunque es importante destacar, que en la mayoría de las comunidades, una buena parte de sus habitantes son de migraciones internas recientes (principios de los años 90), provenientes de Nandaime y occidente del país (León y Chinandega)

Otro elemento encontrado durante la visita es la visión positiva y de enorme esperanza en sus habitantes, como producto de las recientes políticas gubernamentales, pese a que reconocen que falto mucho por hacer. Don Benedicto Francisco Vanegas de la comunidad de Tirurí, refrenda esta visión, cuando reconoce la fluidez de la comunicación entre las comunidades y el gobierno local (la Alcaldía) atendiendo los problemas de sus habitantes: "hay buena relación con la alcaldía y uno de los logros mayores que hemos obtenido en los últimos años es la construcción de la carretera adoquinada. Aunque siguen habiendo algunos problemas en cuanto a atenciones urgentes...".
Por otro lado, la ansiedad por migrar de manera permanenteo portiempos prolongado ha disminuido de manera drástica, según lo expresado por los entrevistados, quienes afirman que ahora van por dos o tres meses, según dure el período de cosecha. Una forma de medir esta reducción en la movilidad de los habitantes, fue observar durante la gira de campo, la presencia de todos o la mayoría de los miembros de las familias en sus casas.

Las mejoras en el acceso a fuentes de trabajo, financiamiento, y planes de atención integral gubernamentales, han propiciado que los habitantes de esta zona tengan menos motivos para viajar por razones de trabajo, a los poblados vecinos del otro lado de la frontera. Aquellos que lo hacen por tradición de trabajo (temporal), en campos de naranja $\mathrm{u}$ otras plantaciones costarricenses van disminuyendo, aunque es importante recalcar, que este flujo migratorio de tipo laboral, no desaparecerá por las relaciones y/o lazos culturales inter fronterizos.

El arraigo en sus localidades como producto de acciones afirmativas de políticas públicas y la confianza en el actual gobierno (en su mayoría), son reforzada con otras acciones sociales (agencia), como la creación de infraestructura comunicacional que los integra a la nación, instalación de señales de telefonía celular, luz eléctrica y la ampliación de las señales de radio y televisión. La mayoría de los entrevistados coincidieron en señalar como un logro importante en materia de atención del Estado y el gobierno, la instalación de redes de señales telefónicas. Ya no necesitan comprar (aunque existen zonas donde la señal no llega o es débil) servicios de telefonía celular costarricense para comunicarse con familiares en Nicaragua.

Otro tema sobre el cual giró las entrevistas, fue la indagación acerca de los medios de comunicación utilizados por los pobladores para informarse de lo que ocurre en el interior del país. La totalidad de los entrevistados coincidieron que la radio es el medio más usado para estar informado del acontecer nacional. Las señales de estaciones de radios 
nicaragüenses más escuchadas provienen de Rivas, Nandaime, Granada, Jinotepe, San Carlos y Managua. De manera particular, las estaciones radiales de la Capital son las más sintonizadas y escuchadas, entre ellas: Radio Ya, La Primerísima, Radio Sandino, Radio Rica (evangélica) y Radio Corporación.

Los habitantes de las comunidades en estudio, en su mayoría son evangélicos. Los católicos son minorías, prueba de ello es que durante la visita de campo se encontró una única capilla de esta orientación religiosa (católicos), mientras que de las denominaciones llamadas "evangélicos" (protestantes), tenían una o dos capillas en cada una de estas comunidades. Eso explica en parte la audiencia de la radio Rica, cuyo perfil es de orientación evangélica. Pero la radio más escuchada en la comunidad, expresada por los entrevistados, es la Radio Ya. El programa más sintonizado de esta emisora es el "noticiero Ya". Radio la Primerísima y la Sandino, también la sintonizan por razones de información y para disfrutar de la música popular (esto se constatará en la encuesta realizada).

La televisión y los periódicos son medios que tienen poco arraigo en el quehacer cotidiano cultural y social de los pobladores. Entre las explicaciones provisionales que se pueden aventurar en esta investigación exploratoria, está -en el caso de la televisión-- el acceso a la energía eléctrica y las condiciones materiales de vida, vinculado a los ingresos. En las comunidades donde encontramos receptores de televisión, sus propietarios aseguraron que hasta hace pocos años los canales nacionales son captados en sus aparatos. Antes de eso, los canales costarricenses eran los únicos que se captaban.

Son poco los canales nacionales que se sintonizan, siendo una minoría la que disfruta de la recreación o distracción de estos medios. Los canales captados en las comunidades en estudio son: 4, 8, 9 y 10 y 11. Como se ha señalado, las comunidades donde tienen aparatos de televisión, son aquellas donde existe luz eléctrica de manera permanente. Los programas que con mayor frecuencia se sintonizan son: en el caso del canal 8 y 10 noticieros (destaca la nota roja); el canal 4 por los temas políticos y aquella información oficial relacionadas con proyectos sociales o campañas nacionales educativas o sanitarias. Los canales 9 y 11 son sintonizados por los programas infantiles, telenovelas (igualmente sintoniza el canal 10) y otros programas de adolescentes.

En el caso de los periódicos no se leen porque no llegan o no están dentro de la ruta (o prioridad) de distribución de estas empresas. La única forma de leer un periódico, de vez en cuando -expresaron los entrevistados que afirmaron leer periódicos-era cuando iban a Rivas. Por tal razón, estos medios no tienen ninguna incidencia en las prácticas discursivas cotidianas de los habitantes, como si lo tienen las radios y la televisión en menor medida.

Esta descripción acerca del acceso y consumo de información de las comunidades en estudio, permite establecer algunos ejes de trabajo acerca de la imagen del sujeto de frontera, o viviendo en espacios fronterizos. Por medio de esta radiografía se puede establecer comparaciones con los modelos discursivos y las representaciones que de estos espacios y de sus pobladores, hacen el resto de los habitantes del interior del país $\mathrm{y}$ de los niveles de influencia que tienen las empresas mediáticas (y sus expresiones escritas o audiovisuales) en uno y en otros con fines auto referenciales.

Ante una pregunta hecha durante las entrevistas acerca de su identidad, todos expresaron sin ninguna duda su nacionalidad nicaragüense y su arraigo a una tierra y un lago, el cual les da su condición de habitante de esta zona. No es la frontera la que modela su identidad sino su condición lacustre, la cultura pesquera, pese a que esta especie es escasa y la vida vinculada al lago se vaya extinguiendo. De igual manera, el hecho que algunos de ellos expresaran su doble nacionalidad (nica-costarricense) su preferencia es vivir en esa franja angosta de 
tierra que limita al vecino del sur y el lago Cocibolca.

\section{Los medios y la construcción del "espacio peligroso"}

La comunicación en nuestras sociedades, como lo expresa Enrique Bustamante en el prólogo al libro de Armand y Michéle Mattelart, Pensar sobre los medios de comunicación. Comunicación y crítica social (1988) "por sus estrechos lazos con todos los dominios sociales, nos acerca a otros muchos fenómenos importantes de nuestra época" (p.14). En este sentido, los medios de comunicación social tienen una enorme importancia en la construcción de prácticas discursivas, de sujetos colectivos vinculados a una comunidad o grupos sociales.

Si partimos de la idea expresada por Rivadeneira Prada (1995) donde afirma que la relación social del hombre solo es posible a través de la comunicación" (p.32), podemos expresar, siguiendo a Halbwachs (2011), que la memoria de cada individuo está mediada por la sociedad y que su pensamiento está condicionado para las prácticas y vivencias cotidianas, que no es más que la "memoria colectiva" o social.

Por otro lado, es interesante el análisis de Iuri Lotman, rescatado por Silva Echeto (2006) acerca del concepto de frontera visto de las representaciones literarias, como la "frontera de la simiósfera", donde establece los límites entre lo extra semiótico (como lo externo) --no sin conflicto-con lo intra semiótico (interno). Para este autor, señala Silva Echeto,

...la frontera es un mecanismo bilingüe que traduce los textos externos al lenguaje interno de ella y a la inversa. La frontera de la semiósfera filtra los mensajes externos y los traduce al lenguaje propio, así como convierte los no discursos externos en discursos (p.4).

Es la conversión de los símbolos y mensajes que viene de "afuera" (como lo no vinculado socialmente y primariamente con un grupo o comunidad en particular) en discursos digeridos internamente por la comunidad. Esa condición se expresa cuando se leen los periódicos de la capital, repetidos por las radios, acerca de los espacios socio-culturales de frontera, articulados por un discurso discriminatorio y violento de los "otros" considerados "externos".

La revisión de las noticias relacionadas con los espacios de la frontera sur (Cárdenas) publicadas en El Nuevo Diario y La Prensa, los dos únicos medios escritos nacionales, construyen un discurso cuyas representaciones de esta zona de frontera, es todo menos una zona de paz, de encuentro y de vida. Basta pasar revista de los titulares, que en su mayoría dan a conocer conflictos y/o violencia: "Incautan 140 kilos de cocaína en frontera Costa Rica Nicaragua" (END 28-01-14); "Nicaragua debe amojonar frontera"(END 26-02-14); “Ticos reforzarán vigilancia en frontera con Nicaragua" (END 07-01-11): "Diputado pide a Nicaragua y Honduras resolver conflicto de tierra" (END 13-11-13).

Los titulares de los diarios reproducidas por la radio y la televisión, construyen un discurso "negativo", como lo sugiere Martínez en su estudio sobre Tijuana, ciudad fronteriza con México. Si bien, los pobladores de estos espacios de frontera nicaragüense no se identifican, ni sienten ser sujetos "peligrosos" o "distintos" al resto de habitantes del país, las representaciones construidas a partir de las informaciones periodísticas, internalizadas por la memoria colectiva de los habitantes del país, modelan un lugar inseguro, poroso para la delincuencia, y por tanto violento.

Las representaciones de unos espacios de frontera sin orden ni ley, ha sido alimentada por un discurso donde el poder juega un papel crucial, sin la cual no se puede comprender la accidentada explicación de la construcción del Estado Nación (Granados, Documento de estudio). Para Manuel Ortiz Marín (2006) tanto las instituciones estatales y los medios de comunicación, mantienen 
una disputa cómplice, porque además de disputarse el poder de influencia o dominación, ambos convergen en intereses "estratégicos", dictados por el modelo de dominación: "La lucha por la dominación provienen de la cultura dominante y se manifiesta en la cotidianidad mediática, por imponer una visión simbólica de la realidad social conforme a sus intereses particulares" (p.22).

Los avatares por el establecimiento de un estado y la construcción del estado-nación en el siglo XIX, tuvo como retos, la defensa de los límites territoriales con sus vecinos, en una lucha que no sólo fue legal sino militar. Para José González Tejada, esta concepción moderna del Estado deja claro que:

...en la formación y el mantenimiento del Estado nación se cuenta con una base cultural compartida que permite la construcción de un marco consensual de sus habitantes. Sin embargo, los Estados nacionales recurren a la violencia y a la imposición en muchas ocasiones porque se ven obligados a establecer el monopolio de la fuerza sobre su territorio. Hacia el centro la identidad nacional es más firme y está más establecida. Al alejarse del núcleo geográfico y político se van dando otras muchas variaciones y combinaciones que tratan de establecer su identidad propia ante el centro, de modo que en la frontera este problema se agrava por el roce constante que se tiene con otras culturas y comunidades (p.82).

Concepción que en la actualidad está siendo cuestionada por paradigmas que sustentan concepciones de globalización o posmodernas que ven en estos espacios, más que límites, procesos pluriculturales, identitarios de cooperación e intercambio (lo que Granados y otros autores llama relaciones transfronterizas). En este sentido, Jorge Eduardo Brenna B. (2010) señala:

Las fronteras ya no son sólo los escenarios en los que se disputan los valores nacionales y se defiende la soberanía. Hoy ocurren un sinnúmero de fenómenos en ellas, transformaciones de todo tipo: económicas, sociales, políticas pero sobre todo culturales, mismas que trascienden los límites de las dinámicas propias de los estados nacionales. La bidimensionalidad identitaria (nosotros y los otros) presente en toda frontera nacional ha cedido su lugar a una multidimensionalidad identitaria (diversos nosotros) detrás de una dinámica transfronteriza que constituye, crea y recrea a las comunidades pluriculturales que se forman en las fronteras en un ambiente potencialmente conflictivo (p.274).

Por último, si bien es cierto en la actualidad ha mejorado la atención del Estado y el gobierno a las comunidades fronterizas, la debilidad del estado-nación y la ausencia de políticas públicas que aborden de manera integral, con planes a mediano y largo plazo, es una asignatura pendiente. Hay que retomar el reto que plantea Brenna B.: “...de encontrar fórmulas políticas democráticas y sobre todo configurar una cultura politica fronteriza para garantizar el diálogo y la negociación de los espacios vitales para reproducir las comunidades culturales y garantizar la convivencia pacífica en estos espacios tan peculiares" (p. 274).

\section{Conclusiones}

$\mathrm{Si}$ bien los habitantes que viven en localidades cercanas a la frontera hoy día se sienten nicaragüenses, igual que el resto de los habitantes del país, la visión que genera el discurso mediático construye un tipo de representación "negativa" de estos espacios. Los medios de comunicación y las instituciones públicas y privadas no abonan en nada para cambiar la imagen negativa dibujada por los discursos elaborados y difundidos desde los centros de poder cultural y políticos del país.

Los habitantes de esta zona de frontera (Cárdenas) tienen una visión positiva y llena de esperanza y de progreso, alimentada 
por políticas estatales y una gestión cercana (dialogante) que promueve el gobierno local. Ellos consideran que falta mucho por hacer pero confían en que los proyectos más urgentes: luz eléctrica, agua potable, mejoramiento de las vías de comunicación, créditos, creación de fuentes de empleo, salud y educación se mejoren y amplíen.

De igual manera, a nivel central falta definir políticas nacionales que atiendan de forma especial el tema de las fronteras y su complejidad, tomando en cuenta la acción afirmativa y la agencia entre sus pobladores. Superar la visión moderna, positivista en que se ha sustentado la explicación de los espacios de frontera, en los centros de toma de decisiones (los poderes centrales) y en las academias con el fin de pensar en una integración transnacional, es un aspecto sobre lo que se debe de trabajar más.

\section{Referencias bibliográficas}

Arias Olave, D. A. (2013) Avances en la caracterización histórica y socieconómica de las relaciones fronterizas en la región Putumayo y Sucumbíos (tesis doctoral). Universidad Nacional de Colombia, sede Amazonía.

Bello de Arellano, M. E. \& Vilera de jirón, A. (2000) La relación sujeto, discurso y poder. La biopolítica, las fronteras y las relaciones internacionales. Una aproximación a los planteamientos de Rafael Parada. Aldea Mundo. V (9), pp. 73-78.

Caballero Santos S. y Tabernero Martin, C. (2015).Delonacionalalotransfronterizo. Resistencia a la estatalidad en África y Latinoamericana. Iconos, Revista de Ciencias Sociales. 51, pp. 11-130. DOI: 51.2015.1476.

Granados, C. (2001). La cooperación transfronteriza en Centroamérica: ¿tregua o nueva visión de soberanía) Conferencia dictada en el marco del Seminario Frontera y Migración. Instituto de Historia de Nicaragua y Centroamérica (IHNCA-UCA).
Managua. Nicaragua.

González Tejada, J. (2004). La línea, la frontera y la modernidad. Estudios Fronterizos $\mathrm{V}(10)$, pp. 73-90.

Halbwachs, M. (2011) La memoria colectiva. Buenos Aires: Miño y Dávila Editores.

Mendoza Pérez, C. (2007) El espacio fronterizo en la articulación de espacios sociales trasnacionales: reflexión teórica y apuntes empíricos. Papeles de Población, (13) 53, pp. 103-135.

Martínez, S.M. (2011) Espacio fronterizo: La Tijuana de Santitos y Peregrinos de Aztlán. Hipertexto, 14, Pp. 95-107.

Mattelart, A y M. (1988) Pensar sobre los medios de comunicación. Comunicación y crítica social. San José: Departamento Ecuménico de Investigaciones.

Mejía M, L., Fermín de Mejía I \& Alcántara A. J, (2013) Conceptualización de espacio fronterizo y delimitación de las áreas de estudio. Consultado 16 de agosto 2015 de la www: http://ance.msinfo. info/bases/biblo/texto/libros / SI.1987.T.I.b.3.pdf.

Rivadeneira Prada, R. (1995). La opinión pública: análisis estructura y métodos para su estudio. México: Trillas.

Silva Echeto, V. (2002) Los medios de comunicación, las fronteras y los feminismos. Consultado 16 de agosto 2015: http://www.escritorasyescrituras. com/revista-detalle.php/1/7/los-mediosde-comunicacin-las-fronteras-y-los feminismos?PHPSESSID=67d7a09d0cf76b 6a34b748f72b175259

Toussaint, F, y Navarro, R. (1991) Frontera Norte: estructura de poder y medios de comunicación. Estudios sobre las Culturas Contemporáneas, IV (11), pp. 131-168.

Vargas Hernández, J.G.(2005). El mercado transfronterizo de servicios de salud privados entre Tijuana, México y San Diego, Estados Unidos Revista de Economía Mundial. 13, 165-184.

\section{Entrevista}

Vanegas, B. F. (8 de julio 2015). Comunidad de Tirurí (M. Ayerdis, entrevistador) 